\title{
Super-resolved Ptychographic Imaging
}

\section{Andrew Maiden}

Dept. of Electronic and Electrical Engineering, University of Sheffield, Sheffield, UK.

Ptychography is a form of Coherent Diffractive Imaging (CDI) in which a localized illumination function (or probe) is sent to a grid of positions on a sample and a detector records a diffraction pattern at each position. Setting the pitch of the position grid smaller than the size of the probe ensures that most points on the sample are illuminated multiple times, whilst structure in the probe ensures that diversity is introduced into the recorded data: each point on the sample is illuminated multiple times in multiple ways. The resulting set of diffraction patterns have been shown to be relatively straightforward to invert to give an image of the sample, quantitative in both amplitude and phase. But in addition, the diversity in a ptychographic data set also encodes a panoply of information that can be processed to yield an image of the probe [1-3], the coherent modes of the probe when it is only partially coherent [4] or of multiple wavelengths [5], corrections to errors in the measurement of the grid of positions through which the probe was scanned [1,6-8], 3D images [9,10], and super-resolved images [11].

Lateral super-resolution is achieved in ptychography by extrapolating diffraction data outside of the numerical aperture of the recording system. Figure 1 gives an example from the visible light regime. Figure la shows the experimental setup - a highly structured illuminating probe is diffracted by a transmissive sample and forms a diffraction pattern at the plane of a detector. The detector has a limited Numerical Aperture (NA): diffraction data outside of this NA, corresponding to the finest features on the sample, are lost and so the image reconstructed from this data is limited in resolution. Figure $1 \mathrm{~b}$ shows an image of a resolution target reconstructed from the NA-limited diffraction patterns, the extent of which is demarked by the red box in Figure 1c. During the reconstruction process however, it is possible to extrapolate well beyond the confines of the detector NA [11]. Figure 1d shows the resolution target reconstructed from the same diffraction data when the extrapolating algorithm is used: the algorithm recovers all of the diffraction data between the red square and the blue circle in Figure 1c.

Super-resolution ptychography is only one of a number of avenues of current research into the rich ptychographic dataset. It is particularly relevant to the visible light regime, where it can offer high image resolutions at large working distances. However it may also find use in the scanning transmission electron microscope, where it promises to allow the extrapolation of dark-field diffraction from measurement of the bright-field diffraction pattern [12,13]. 


\section{References:}

[1] M Guizar-Sicairos and JR Fienup, Opt. Express 16 (2008), p. 7264.

[2] P Thibault et al, Science 321 (2008), p. 379.

[3] AM Maiden and JM Rodenburg, Ultramicroscopy 109 (2009), p. 1256.

[4] P Thibault and A Menzel, Nature 494 (2013), p. 68.

[5] D Batey et al, Ultramicroscopy 138 (2014), p. 13.

[6] AM Maiden et al, Ulramicroscopy 120 (2012), p. 64.

[7] M Beckers et al, Ultramicroscopy 126 (2013), p. 44.

[8] F Zhang et al, Opt. Express 21 (2013), p.13592.

[9] AM Maiden et al, JOSA 29 (2012), p. 1606.

[10] A Suzuki et al, PRL 112 (2014), 053903.

[11] AM Maiden et al, JOSA 28 (2011), p. 604.

[12] TB Edo et al, Proc. SPIE 7729 (2010), 77291H.

[13] This work was funded in part by EPSRC Grant No. EP/E034055/1 and by Phase Focus Ltd.



Figure 1. Super-resolved ptychography. (a) Experimental setup: a microscope objective forms a $\sim 50 \mu \mathrm{m}$ spot on a specimen and a CCD records the diffraction pattern generated downstream. The CCD has a smaller NA than the illumination optics (blue lines) and does not capture the full extent of the diffraction data (red lines). (b) A conventional ptychographic reconstruction using the data demarked by the red square in (c) produces only a moderate-resolution image. (d) The super-resolution algorithm extrapolates the diffraction patterns to the extent shown by the blue box in (c) to produce an image with a sub-micron resolution. Scale bar $10 \mu \mathrm{m}$. 